\title{
Correlative Microscopy of the Caulobacter crescentus Flagellum Reveals How Changes to the Flagellin Protein Sequence Regulate Structure and Function.
}

\author{
Elizabeth R. Wright ${ }^{1}$, Rebecca S. Dillard ${ }^{1}$ and Conrad W. Shebelut ${ }^{1,2}$ \\ 1. Department of Pediatrics, Emory University School of Medicine, and Children's Healthcare of \\ Atlanta, Atlanta, Georgia, USA. \\ 2. University of Virginia School of Medicine, Charlottesville, Virginia, USA.
}

The bacterial flagellum is a complex propeller and is composed of three major units: the motor (basal body), the hook, and the filament. As a whole, the components work together to drive the bacterium through its environment. A number of studies of many bacterial species, including Caulobacter and Vibrio species, indicate that flagellar filaments are assembled from several flagellin proteins as opposed to a singular flagellin. Many of the flagellins are differentially regulated, redundant in molecular weight, and function [1]. While there may be some redundancy, there appears to be evidence that a single flagellin is essential for flagellar filament synthesis and motility. Caulobacter crescentus expresses six flagellin proteins, and all the flagellins are present along the length of the flagellar filament [1]. In this structural study of the Caulobacter flagellum, we sought to ascertain which flagellin was essential and determine the high-resolution structure of both the full-length flagellum and the flagellin protein. We used a combination of mutagenesis experiments, fluorescence microscopy, and cryo-electron microscopy (cryo-EM) to generate and identify the appropriate mutants for structure determination.

Caulobacter crescentus flagellum labeling was done using Alexa Fluor $495 \mathrm{C}_{5}$ Maleimide (AF495-mal) (ThermoFisher Scientific). C. crescentus cultures were grown to mid-log phase before labeling. Labeled cultures were gently centrifuged and washed once with PYE before being concentrated. Cells were imaged using phase contrast microscopy, while the labeled flagella were imaged using fluorescence microscopy.

Negative stain TEM of the bacteria and isolated flagella followed standard procedures. Briefly, 400 mesh carbon-formvar-coated copper grids were glow discharged for 30-60 seconds. Four $\mu \mathrm{L}$ of the sample was applied to the grid and allowed to adsorb for 1 min before staining. Negatively stained specimens were imaged on a JEOL JEM- 1400 TEM (JEOL Ltd., Tokyo, Japan) equipped with a LaB 6 filament and operated at an accelerating voltage of $120 \mathrm{kV}$. Images were digitally captured on a Gatan US1000 CCD camera (Gatan, Pleasanton, CA, USA).

Aliquots of bacterial cells were flash frozen onto glow-discharged Quantifoil carbon grids in liquid ethane with either a ThermoFisher (FEI) Mark III Vitrobot or a Leica EM-GP. Cryo-EM and cryo-ET data collection was performed with a JEOL JEM-2200FS $200 \mathrm{kV}$ FEG-TEM equipped with an incolumn energy filter ( $s$ lit width $20 \mathrm{eV}$ ), a cryo-transfer specimen holder (Model 914, Gatan), a 4k x 4k Gatan Ultrascan CCD camera, and a Direct Electron DE-20 direct electron detector. Images were acquired with a pixel size ranging from 0.614 to $0.294 \mathrm{~nm}$ on the specimen. For tilt series, a total electron dose of $\sim 120 \mathrm{e}^{-} / \AA^{2}$ was fractionated over tilt series ranging from $-62^{\circ}$ to $+62^{\circ}$. Tilt series images were taken automatically with $2^{\circ}$ tilt increments by using Serial EM [2].

In our study, we have been able to generate a series of mutant Caulobacter strains, each able to synthesize and assemble straight flagella. We used a combination of imaging methods to validate the 
presence of straight flagella extending from the cell body and upon isolation (Figure 1).

References:

[1] A Faulds-Pain, et al. Journal of Bacteriology 193(11) (2011), p. 2695.

[2] JR Kremer, DN Mastronarde, and JR McIntosh, Journal of Structural Biology 116(1) (1996), p. 71.

[3] This research was supported by funds from Emory University, Children's Healthcare of Atlanta, the Emory Center for AIDS Research, the Georgia Research Alliance, Human Frontiers Science Program, National Institutes of Health (R01GM104540 and R01GM104540-03S1), and the National Science Foundation (0923395) to E.R.W, and S10 RR025679 to P.W.S. All EM data was collected at the Emory University Robert P. Apkarian Integrated Electron Microscopy Core.
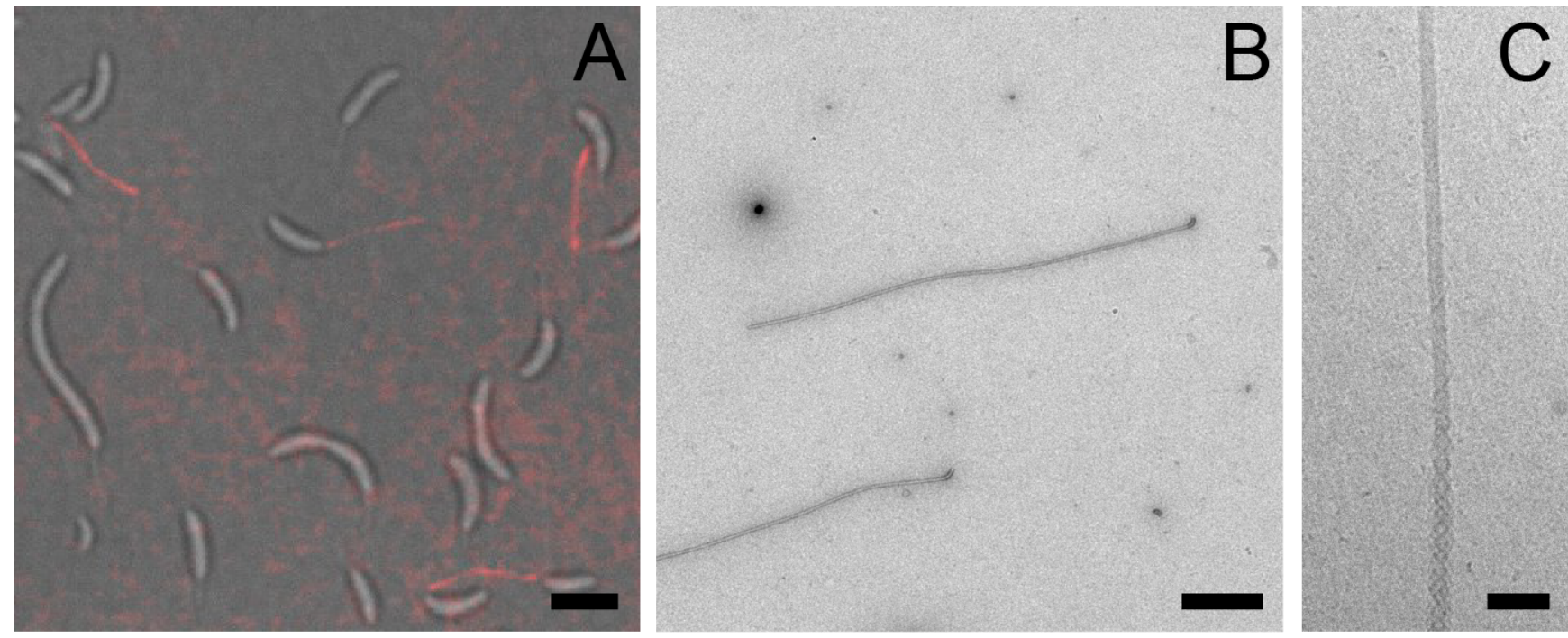

Figure 1. Imaging studies of the Caulobacter crescentus flagellum. (A) Fluorescence microscopy of Alexa Fluor $495 \mathrm{C}_{5}$ Maleimide-labeled flagella, cell bodies were imaged via phase contrast. Note that the flagellar filaments are straight. (B) Negative stain TEM imaging of isolated flagella from the Caulobacter strain identified in (A). (C) Cryo-electron microscopy (cryo-EM) 2D image of an isolated Caulobacter flagellum. Scale bars are $2 \mu \mathrm{m}, 500 \mathrm{~nm}$, and $50 \mathrm{~nm}$. 